\title{
$H \alpha$ Kinematics of Tidal Tails in Interacting Systems: Projection Effects and Dark Matter in TDGs
}

\author{
Philippe Amram ${ }^{1}$, Fréderic Bournaud ${ }^{2,3}$, Pierre-Alain Duc ${ }^{2}$ \\ 1 Observatoire de Marseille, France \\ 2 CEA, Saclay, France \\ 3 Observatoire de Paris, France
}

\begin{abstract}
Several interacting systems exhibit at the tip of their long tidal tails massive condensations of atomic hydrogen, which may be the progenitors of Tidal Dwarf Galaxies. Because, quite often, these tails are observed edge-on, projection effects have been claimed to account for the large HI column densities measured there. Here we show that determining the velocity field all along the tidal features, one may disentangle projection effects along the line of view from real bound structures. Due to its large field of view, high spectral and 2D spatial resolutions, Fabry-Perot observations of the ionized gas are well adapted to detect a kinematical signature of either streaming motions along a bent tidal tail or of infalling/rotating material associated with a forming TDG. Spectroscopic observations also allow to measure the dynamical masses of the TDGs that are already relaxed and check their dark matter content.
\end{abstract}

\section{Introduction: the kinematics of tidal tails}

The most impressive and surely most studied interacting systems, such as the Antennae galaxies, exhibit long optical tidal tails that may extend up to 100 kpc. HI observations of such colliding galaxies have shown that the stellar tails have a gaseous counterpart that is usually even more prominent and contain a large fraction of the total atomic gas present in the system. In a few interacting galaxies, the HI tidal tails exhibit some gas concentrations that have apparent masses of up to few $10^{9}$ solar masses. Those are the progenitors of the Tidal dwarf galaxies (TDGs), at least the more massive ones. They are typically found at the tip of the optical tidal tails at distances between 30 and $100 \mathrm{kpc}$ from the merging disks. They might be as massive as the Magellanic Clouds, and on top of being rich in atomic hydrogen gas (Duc et al. 2000), they contain high quantities of molecular gas (Braine et al. 2001, and his contribution in this volume) and form stars with a rate as high as in blue compact dwarf galaxies (Duc \& Mirabel 1998). The amount of stellar and gaseous material in several TDGs suggests that they are gravitationally bound, although we do not have yet conclusive observational evidence of such a self-gravitating TDG, kinematically independent from its host tidal tail. Moreover if dark matter is made of collisionless material distributed in a large halo, it should not be ejected with tidal stellar and gaseous debris pulled out from colliding disks (Barnes \& Hern- 
quist, 1992). In that case, TDGs should not contain a significant amount of DM whereas ordinary dwarf galaxies seem to possess a lot of it. This can be checked by determining the dynamical masses of TDGs.

However, the existence of the most massive Tidal Dwarf Galaxies or even of their gaseous progenitors as independent entities has been challenged (see Hibbard et al., in this volume). Indeed, an apparent accumulation of tidal material could in reality be the result of a projection effect. In the $3 \mathrm{D}$ space, tidal tails are curved. Seen edge-on, they appear as linear structures and may present at their tip fake mass concentrations due to the presence of projected material along the line of sight. Using numerical simulations, we have shown that such projection effects have a kinematical signature (Bournaud et al. 2004). Indeed, as shown in Fig. 1, the large-scale velocity gradient corresponding to the streaming motions along the expanding tails changes its sign before their apparent extremity whenever a projection effect exists.

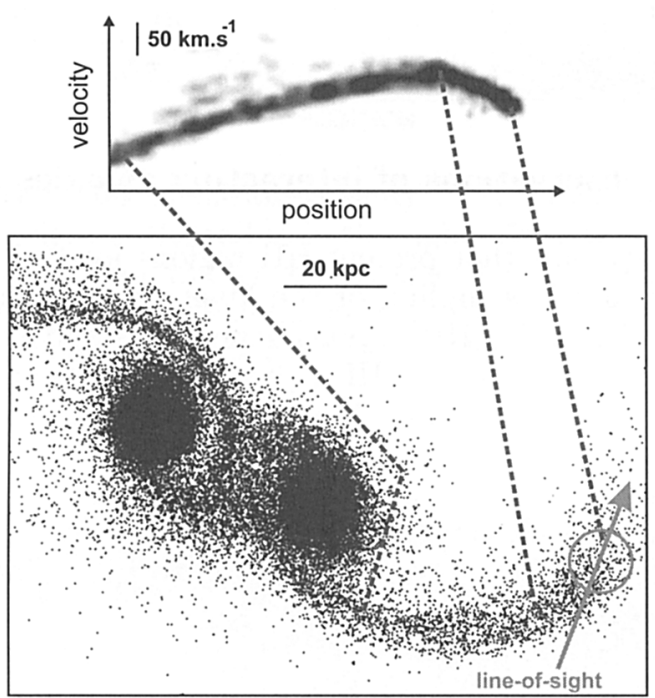

Figure 1. Numerical simulations of two interacting galaxies. The kinematics of one of the tidal tails is analyzed as if it were observed edge-on, assuming that the line-of-sight is aligned with its extremity. This projection effect may result in an apparent accumulation of matter at the tip of the observed tail. The position-velocity diagram derived from this simulation shows a velocity gradient, mainly related to streaming motions along the tail. The sign of this gradient changes before the extremity of the tidal tail, a result which is obtained whenever a part of the tail is aligned with the line-of-sight. Conversely, a velocity gradient that has a constant sign rules out the presence of a projection effect. In addition to the large-scale motions, each TDG or gravitational clump can have an inner velocity gradient, related to its own dynamics. 
Such a study requires observations with an instrument enabling the access to both the large-scale and small-scale dynamical structures. Synthesis arrays of radiotelescopes allow to probe the largest ones but do not have enough sensitivity to reach the $1^{\prime \prime}$ spatial resolution required to sample correctly the nearby TDGs progenitors. Because of their low surface brightness, a direct study of the stellar kinematics of tidal features is at the limits of today's telescopes and detectors. The internal dynamics can be more easily approached in the ionized gas component through spectroscopic observations of the emission lines. Slit '1D' spectroscopy already provides some information (see Weilbacher et al. 2003) but is largely insufficient given the complex morphology of colliding galaxies. The Fabry-Perot technique appears as an ideal tool as it combines an integral-field capability, a high spatial and spectral resolution and a large field of view. We present here Fabry-Perot observations of several interacting systems where TDG candidates had been previously identified. Observations were carried out at the European Southern Observatory $3.6 \mathrm{~m}$ telescope and at the Canada-France-Hawaii $3.6 \mathrm{~m}$ telescope. The pixel size on the sky varies from 0.86 to 0.91 arcsec; the FOV from 170 " $\times 170$ " to 440 " $\times 440$ " and the velocity sampling from 10 to $16 \mathrm{~km} . \mathrm{s}^{-1}$. The data reduction procedure has been extensively described (Amram et al. 1998 and references therein).

\section{Fabry-Perot observations of interacting galaxies.}

We have observed systems that present HII regions located all along the tidal tails, which ensures a fair sampling of the kinematics of the tidal structures. The most active and massive HII regions generally stand at their extremities, and correspond to accumulations of $\mathrm{HI}$ with typical masses of $10^{9} \mathrm{M}_{\odot}$, and are thus massive TDG candidates. Two kind of kinematical features are observed in the tidal debris:

- large-scale velocity gradients resulting from streaming motions and the projection of velocities along the line-of-sight

- small-scale velocity gradients, possibly related to the collapse or the rotation of decoupled objects in the tails.

\section{Streaming motions and projection effects}

We present here our results for three systems (more in Bournaud et al., 2004) where the presence of massive TDGs had previously been claimed: IC 1182, a merger in the Hercules cluster (see Fig. 2 and van Driel et al., in these proceedings), Arp 105 (NGC 3561, "The guitar"), an interacting system between a spiral and an elliptical (see Fig. 3) and Arp 242 (NGC 4676, "The Mice"), an interacting system between two spirals with prominent tidal tails (see Fig. 4, and Hibbard et al., in this volume).

The tail of one system, IC 1182, shows a large-scale kinematics suggesting that projection effects may play a role, even though the presence of a genuine accumulation of matter, corresponding to a TDG, cannot be excluded.

The shapes of the position-velocity diagrams of the two other systems, Arp 105 and Arp 242 rule out projection effects: the massive condensations observed at the extremity of their tidal tails seem real. This is consistent with 


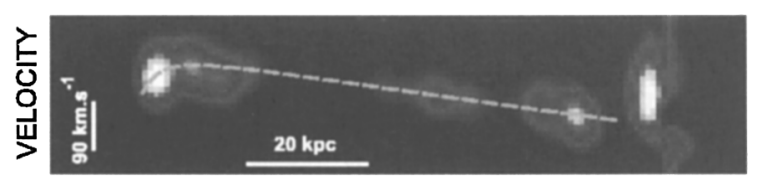

POSITION

Figure 2. IC 1182: Position-velocity diagram along the western tidal tail (see van Driel et al. in these proceedings). Several HII regions are present along the tidal tail, the most intense one being at its end. The velocity gradient changes its sign before the massive TDG candidate, which is consistent with the kinematical signature of a projection effect (see Fig. 1).

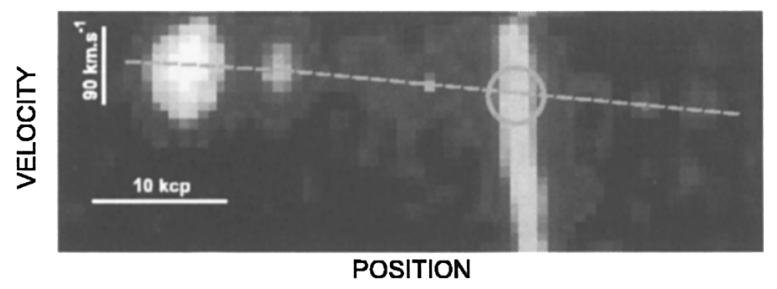

Figure 3. Arp 105: Position-velocity of the southern tidal tail of Arp 105. The origin of the tail is to the right. An HII region decoupled from the elliptical, but hidden by it on this diagram (see Bournaud et al. 2004), is encircled. The large-scale velocity curve is highlighted by the dashed line. It is constant along the tail. According to our numerical simulations, this suggests that the massive TDG candidate located at the extremity is not due to a projection effect.

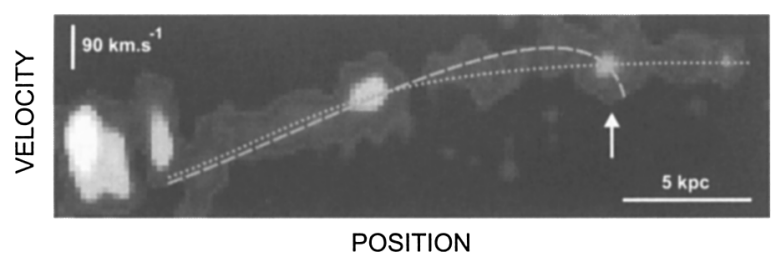

Figure 4. Arp 242: Position-velocity diagram along the northern tidal tail. The origin of the tail is to the left. The position of one massive TDG candidate is indicated by the arrow. The velocity curve may be similar to that expected for a model with a projection effect, i.e. having a change in the sign of the velocity gradient (long-dashed line). However, the kinematics of the last $5 \mathrm{kpc}$ is then not fitted at all. A model with a monotonically increasing velocity (short-dashed line), without any projection effect, seems more robust.

the detection in these TDG candidates of molecular gas probably formed in the dense HI tidal clouds (Braine et al., 2001). 


\section{Inner dynamics of TDGs}

Beside providing information on the large-scale kinematics of tidal tails, our FP observations were useful to study the internal small-scale kinematics of the condensations present along the tidal tails. Velocity gradients of typically 50$100 \mathrm{~km} / \mathrm{s}$ were observed in the tails of Arp 105, IC 1182, and NGC 5291. We best explained them by the local effect of gravity. Because of the higher spectral resolution of the FP instrument, the gradients are generally smaller than those initially measured in earlier observations with long slit spectroscopy.
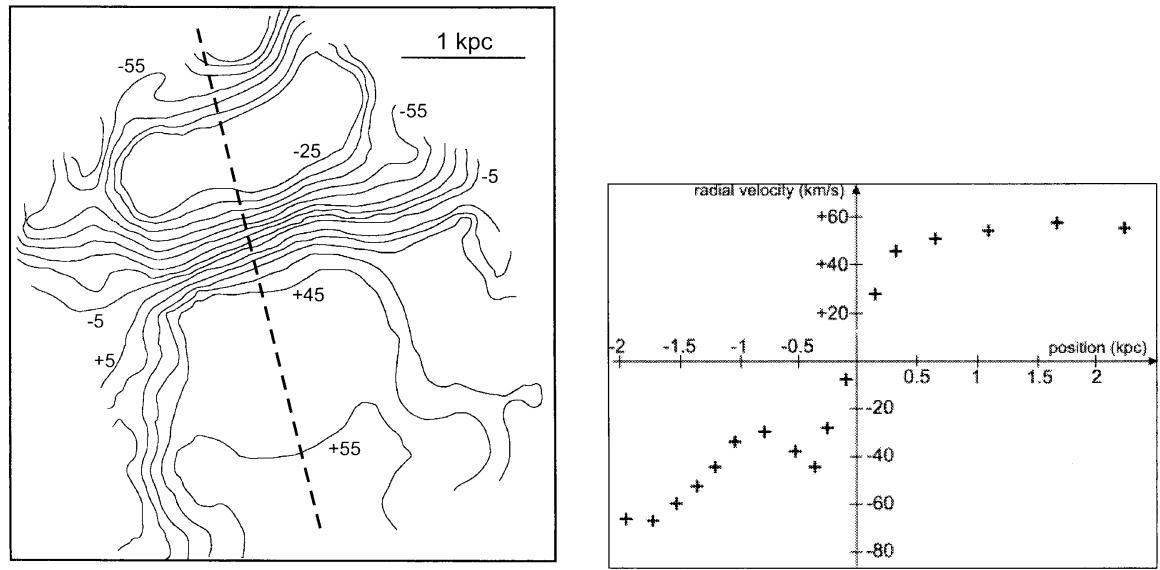

Figure 5. NGC 5291: Left: Isovelocities diagram of the northern TDG candidate. Labels are in $\mathrm{km} . \mathrm{s}^{-1}$. This diagram strongly suggests that the system is already rotating, even though other motions disturb the classical spider shape. This TDG candidate is thus decoupled from the large-scale HI structures and its inner dynamics can be studied. Right: a velocity gradient of $100 \mathrm{~km} . \mathrm{s}^{-1}$, with a disturbed rotation curve, is observed along the dashed line.

The more striking object showing an inner velocity gradients is observed in the northern structures of NGC 5291 ("The SeaShell"). This system is remarkable for the extremely large and massive HI ring surrounding a disturbed lenticular galaxy. Numerous intergalactic HII regions are located towards the HI clumps in the ring (Duc \& Mirabel 1998) $)^{1}$. The northern TDG candidate shows a velocity gradient of $100 \mathrm{~km} . \mathrm{s}^{-1}$ throughout a region of $2.4 \mathrm{kpc}$, and the isovelocity diagram is that of a rotating but disturbed system (see Fig. 5). If we assume an inclination of approximatively 45 degrees (based on the isovelocities diagram and on the shape of the outer $\mathrm{H}_{\alpha}$ isophotes), the deprojected velocity gradient is $140 \mathrm{~km} \cdot \mathrm{s}^{-1}$. Assuming that the TDG is a relaxed rotating system, we find a dynamical mass of $1.410^{9} \mathrm{M}_{\odot}$. We estimate that the contribution

\footnotetext{
${ }^{1}$ Although the origin of the ring is strictly speaking probably not tidal, it was undoubtedly shaped by a past collision, and the small-scale phenomena that occurred in the collisional debris, in particular the onset of star formation, are probably the same as in more typical Tidal Dwarf Galaxies.
} 
due to non-circular motions (that are visible in the isovelocity diagram) is of the same order (Braine et al., 2001). The HI mass of the TDG is $2.610^{9} \mathrm{M}_{\odot}$. Taking into account the additional contribution of the molecular gas and the stars, the visible mass matches the dynamical mass. A straightforward conclusion would be that this system contains no dark matter, as already suggested by Duc \& Mirabel (1998). However, because of our limited spectral resolution, the measure of the dynamical mass is still uncertain and the HI mass may be an upper limit if the HI beam is larger than the clump of HII regions. It is thus not impossible that the visible mass is lower than the dynamical mass and that this TDG contains some dark matter.

\section{Summary and conclusions}

We have shown that the kinematics of tidal tails may be used to check whether the claimed massive TDG candidates observed at their tip are real bound entities or the result of projection effects. With their high spatial resolution, threedimensional Fabry-Perot observations in the $\mathrm{H} \alpha$ line turn out to be well adapted to the problem. Analyzing position-velocity diagrams derived from FP data obtained at the CFHT and at the ESO $3.6 \mathrm{~m}$, we were able to rule our projection effects as the main contributor to the condensations seen in the northern tail of Arp 242 (NGC 4676) and in the southern tail of Arp 105. We cannot exclude that they play a role in the merger IC 1182. The existence of massive entities observed near the tip of several tidal tails is actually supported by the numerical simulations of interacting galaxies by Bournaud, Duc \& Masset (2003) in which tidal objects with comparable masses are formed.

We have also studied the inner kinematics of a few TDG candidates. Several velocity gradients were found. The kinematics of the northern mass concentration of NGC 5291 is consistent with that expected for a self-gravitating rotating object. Its inferred dynamical and luminous mass compare, but higher resolution observations are required to prove that the object does not contain a certain amount of dark matter.

\section{References}

Amram, P., Mendes de Oliveira, C., Boulesteix, J. and Balkowski, C., 1998, A\&A 330, 881

Barnes, J. E. and Hernquist, L., 1992, Nature 715, 360

Bournaud, F., Duc, P.-A. and Masset, F., 2003, A\&A 411, L469

Bournaud, F, Duc, P.-A., Amram, P. and Combes, F., 2004, submitted to A\&A

Braine, J., Duc, P.-A., Lisenfeld, U., Charmandaris, V., Vallejo, O., Leon, S. and Brinks, E., 2001, A\&A 378, 51

Duc, P.-A., \& Mirabel, I.F. 1998, A\&A, 333, 813

Duc, P.-A., Brinks, E., Springel, V., Pichardo, B., Weilbacher, P., and Mirabel, I.F. 2000, AJ 120, 1238

Weilbacher, P. M., Duc, P.-A. and Fritze-v. Alvensleben, U., 2003, A\&A 397, 545 